\title{
Stellar and dust SED modelling of the Whirlpool interacting galaxy system
}

\author{
Erin Mentuch and Christine Wilson \\ Dept. of Physics \& Astronomy, McMaster University, Hamilton, Ontario, L8S 4M1, Canada \\ email: mentuch@physics.mcmaster.ca
}

\begin{abstract}
Some 300-500 Myr ago, the Whirlpool galaxy (NGC 5194/M51a) and its nearby post-starburst galaxy neighbour, NGC 5195/M51b closely interacted, resulting in significant changes to their star formation activity. Both galaxies display colors indicative of enhanced star formation during closest passage, but since then, star formation has ceased in NGC 5195 yet remained ongoing in the spiral NGC 5194. With a wealth of multi-wavelength $(0.2-500 \mu \mathrm{m}$ for this study) observations available, this nearby $(10 \mathrm{Mpc})$ system, whose star formation history is well constrained through optical colors of individual stars and its dynamical history, provides the optimal laboratory to test the relation between dust emission and stellar emission within the fundamental framework of today's stellar population synthesis and dust emission models.
\end{abstract}

Keywords. galaxies: individual (NGC 5194/M51a, NGC 5195/M51b), galaxies: general, galaxies: fundamental parameters, galaxies: stellar content, infrared: galaxies, techniques: photometric

\section{Introduction}

Standard stellar population techniques can tell us how much stellar mass is in a galaxy, its metallicity, the amount of dust extinction and can even shed light on the star formation history within a galaxy. Most dust emission models (Dale \& Helou 2002, Draine et al. 2007) probe the total dust mass and some (Désert et al. 1990, Compiègne et al. 2011) the relative abundance of various dust emission components (eg. polycyclic aromatic hydrocarbons (PAHs), very small grains, big grains). The amount of dust emission observed in a galaxy is tied to both the interstellar radiation field (provided by stellar radiation, in addition to non-stellar sources such as active galactic nuclei) and the abundance of dust grains. How these two processes relate to the stellar populations which both provide the heating and create the dust is not yet well constrained by observations. Fortunately, observations of nearby galaxies in the infrared with the Herschel Space Observatory offer the next step towards the development of fully consistent galaxy population models that can account for dust created and heated by stellar populations, in the context of a galaxy's star formation history.

Observed as part of the Herschel Very Nearby Galaxies Survey (VNGS, P.I. Christine Wilson), we have performed a spatially resolved spectral energy distribution (SED) fitting analysis on the well studied grand design spiral, the Whirlpool galaxy (NGC 5194/M51a) and its post-starburst nearby neighbour, NGC 5195/M51b. While star formation is ubiquitous and ongoing across NGC 5194 as indicated by its $\sim 19600$ HII regions (Lee et al. 2011) and optical colors (Tikhonov et al. 2009), the smaller (in angular extent) neighbour NGC 5195 shows no signs of recent star formation through a deficiency of ionized gas (Thronson et al. 1991). This lack of star formation is supported by evidence for dynamically stable (and thus, unable to form stars) molecular gas (Kohno et al. 2002). An encounter between the two galaxies 300-500 Myr ago is inferred from both kinematic (Salo \& Laurikainen 2000) and hydrodynamic modelling (Dobbs et al. 2010) 


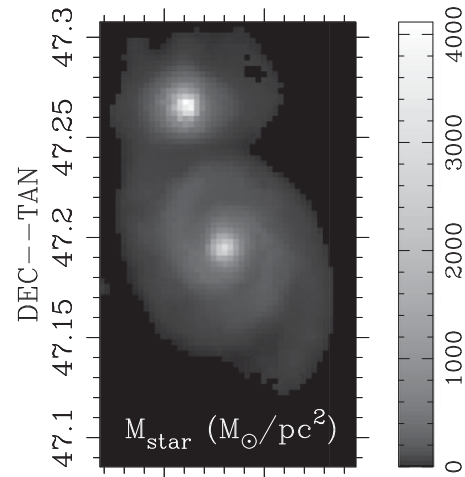

202.5202 .45

$\mathrm{RA}---\mathrm{TAN}$

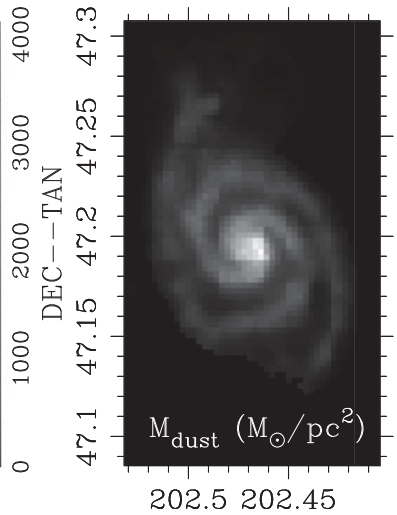

RA-- - TAN

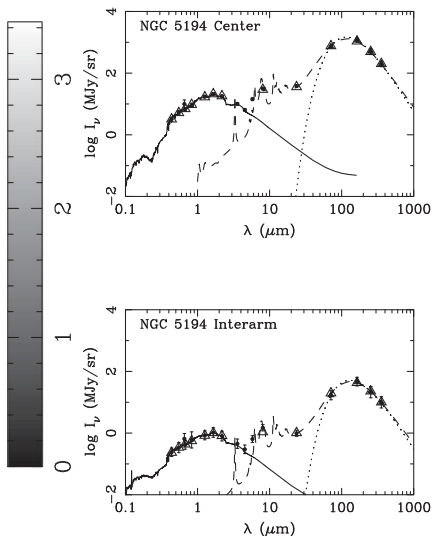

Figure 1. Stellar mass (left) and dust mass (middle) maps of the Whirlpool system. Far right: We plot two example SED fits from the nuclear region of NGC 5194 (top) and the inter arm region of NGC 5194 (bottom). The solid curve is the best fit stellar emission model (PEGASE.2, Fioc \& Rocca-Volmerange 1997) and the dashed curve is the best fit dust emission model from Draine \& Li (2007). For comparison, we also show the best fitting modified greybody model (dotted curve) for both examples.

of the Whirlpool system. In agreement, a recently constructed color-magnitude diagram of its individual stars, measured using HST images, suggests a burst of star formation occurred 390-450 Myr ago in NGC 5195 (Tikhonov et al. 2009). In contrast, this same work posits that NGC 5194 is better described by two episodes of star formation, one at 265-325 Myr and another at 380-450 Myr. Not unexpectedly, this actively star forming disk galaxy cannot be easily described by just a simple stellar population.

As both galaxies display peaks in their star formation during the epoch of closest encounter, how well do SED models retrieve this information? What about the dust emission? How much can be attributed to more evolved stars and how much to more recent star formation? In these proceedings, we highlight the results (to be published in detail in Mentuch et al. 2012, in prep) of our spatially resolved SED fitting analysis of the Whirlpool system in the context of these fundamental questions.

\section{Data and models}

Our analysis includes twenty multi-wavelength images of the Whirlpool system, exploiting the great efforts of a number of large legacy surveys. Our optical $(B, V, R, I$ $\& H \alpha$ ) and mid-infrared (IRAC 3.6, 4.5, 5.6, 8.0 and MIPS $24 \mu \mathrm{m}$ ) observations come from the SINGS survey (Kennicutt et al. 2003). Near-infrared $J, H, K$ imaging is from the 2MASS LGA survey (Jarrett et al. 2003). VNGS's observations with Herschel cover $70,160,250,350$ and $500 \mu \mathrm{m}$. In addition, imaging of the neutral gas is provided by the BIMA SONG survey (Helfer et al.2003) and we have molecular gas observations at CO(10) observations from the Nobeyama $45 \mathrm{~m}$ telescope provided by Koda et al. (2011). All optical and near-IR images are corrected for galactic foreground extinction and matched to a common resolution of FWHM $=28$ " using either gaussian convolutions or in the case of our Herschel observations, kernels from Aniano et al. (2011). At this common resolution and a distance of $8.4 \mathrm{Mpc}$ (Feldmeier et al. 1997), we resolve the galaxies on physical scales of $\sim 1 \mathrm{kpc}$. 
We chose to model stellar and dust emission as separate components using template SED libraries that have both been extensively tested and the parameter space well constrain by observations. Spectral SED libraries were created using standard code from PEGASE.2 (Fioc \& Rocca-Volmerange 1997). Details can be found in Mentuch et al. (2012, in prep), but in brief, our spectral library spans metallicities from 0.005 to 0.04 and the star formation history is modelled as a sum of a continuous star forming population described by a tau exponential with an additional burst of star formation whose amplitude and age are both free parameters. Dust extinction, whose amplitude is an additional parameter, is modelled using a Milky Way extinction law (Pei 1992).

A least-squares fitting algorithm selects the best fit model from our suite of 350000 stellar models to our BVIRJHK images. We use the modelled stellar maps of the galaxy to subtract the contribution of stellar emission from our mid-infrared images in the IRAC 5.6 and $8.0 \mu \mathrm{m}$ bands. These resulting images and our other infrared $(24-500 \mu \mathrm{m})$ images are fit to the two-component emission model of Draine \& Li (2007). In their model, the mid- through far-infrared emission due to PAHs, very small dust grains and carbonaceous dust grains is expressed as a function of the underlying interstellar radiation field (ISRF) comprised of a high energy component surrounding massive star forming regions and then a large scale interstellar medium component with a constant ISRF. The bulk of dust mass is contained in this latter component. In our least-squares fitting procedure, free parameters included the minimum strength ( $U_{\min }$, see Draine et al. 2007 for details) of the radiation field, the fractional contributions of both ISRF components and the fraction of $\mathrm{PAH}$ molecules.

\section{Maps of SED fitted parameters}

A large number of output parameters are yielded from each pixel fit. We generate maps of each parameter using the average of the best fits from a Monte Carlo fitting simulation (see Mentuch et al. 2012, in prep). Here, we highlight some of the results of our fitting which confirm the encounter model and some of the surprises. Figure 1 shows the stellar and dust mass maps in the left and middle panels with examples of our SED fits on the right. The stellar mass map displays a radially smooth distribution with NGC 5194 displaying a typical profile for a spiral galaxy and NGC 5195 showing a steeper profile as an S0. There are some enhancements in stellar mass along the spiral arms, but they are minimal. Coincidentally, both galaxies have very similar stellar masses of $\mathrm{M}_{\star}=(3.62 \pm 0.03) \times 10^{10} \mathrm{M}_{\odot}$ and $\mathrm{M}_{\star}=(2.01 \pm 0.04) \times 10^{10} \mathrm{M}_{\odot}$ for NGC 5194 and NGC 5195, respectively, despite their different morphologies and angular sizes. On the other hand, their dust masses differ by an order of magnitude; $M_{d u s t}=(7.75 \pm 0.02) \times 10^{7} M_{\odot}$ and $\mathrm{M}_{\mathrm{dust}}=(7.67 \pm 0.04) \times 10^{6} \mathrm{M}_{\odot}$ for $\mathrm{NGC} 5194$ and NGC 5195, respectively. It is difficult to speculate whether the difference in the dust-to-stellar mass ratio is primarily due to morphology, the presence of active star formation, or a consequence of the gas fraction in each galaxy prior to interaction. Currently, the gas-to-dust ratio across both galaxies is fairly constant with values in the range of 80-120, typical for spiral galaxies. Figure 2, left panel, shows a mass map of the total gas, traced well by the dust mass surface density shown in black contours.

Reassuringly, the SED fits are consistent with an increased epoch of star formation 300-500 Myr ago. Both galaxies have old stellar populations that have been forming stars continuously (assuming an exponentially declining SFH) for about 8.6 billion years and both had about $10 \pm 6 \%$ of their stellar mass formed in a burst of star formation which occurred $360 \pm 10$ Myr ago. We note here that because of the simplicity of our chosen 

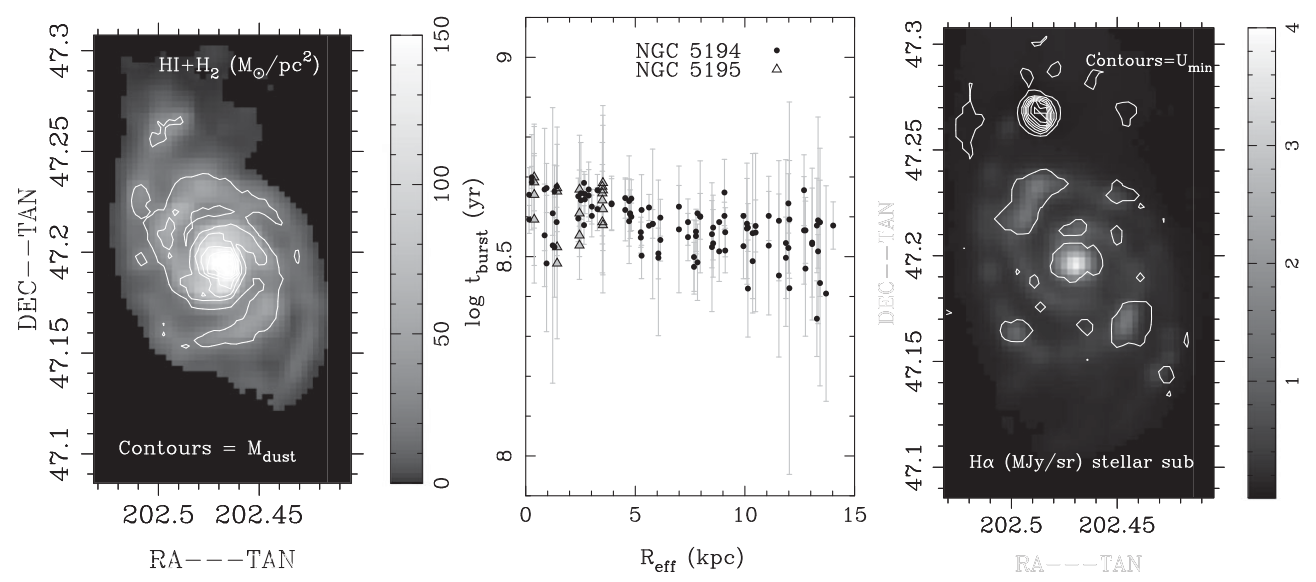

Figure 2. Left: Total gas (molecular $\mathrm{H}_{2}$ from $\mathrm{CO}(1-0)$ plus neutral $\mathrm{HI}$ gas) mass map with dust mass overlaid in contours. Middle: Time since an instantaneous burst of star formation from our SED fitting analysis matches the time of closest approach between the galaxy pair derived from dynamical simulation and colors of individual stars. Here we plot a radial profile of this parameter for each galaxy, NGC 5194 in solid dots and NGC 5195 in open triangles. Right: Map of the stellar subtracted $\mathrm{H} \alpha$ emission with the derived ISRF, quantified as $\mathrm{U}_{\mathrm{m} \text { in }}$ from Draine \& $\operatorname{Li}(2007)$, shown as contours. The highest ISRF is found in the post-starburst galaxy, NGC 5195, where a clear lack of $\mathrm{H} \alpha$ emission is found.

$\mathrm{SFH}$, we do not pick out all of the current star formation occurring in the spiral arms of NGC 5194 in which relatively little stellar mass contributes to the total SED.

One surprising result to come out of our study is the presence of large amounts of dust in the post-starburst galaxy NGC 5195 as is seen in the middle panel of Figure 1. Dust extinction is seen at visible wavelengths extending from one of the spiral arms of NGC 5194 and Tikhonov et al. (2009) confirm that a large population of post-mainsequence stars make up this region. The dust in NGC 5195 is heated by a strong radiation field as indicated by large $U_{\text {min }}$ values (and high dust temperatures), despite a lack of $\mathrm{H} \alpha$ emission (ie. active star formation). Thus, in agreement with Kohno et al. (2002) who found a lack of cold unstable dense gas, but large amounts of stable molecular gas, NGC 5195 appears to be deficient in cold dust but contains a good amount of warm dust.

\section{Acknowledgements}

E. Mentuch would like to thank the IAU organizing committee for the travel grant and acknowledge the continued support for this research provided by the National Science and Engineering Research Council (NSERC) of Canada through her PDF fellowship.

\section{References}

Aniano, G., Draine, B. T., Gordon, K. D., \& Sandstrom, K. 2011, PASP, 123, 1218

Compiègne, M., Verstraete, L., Jones, A., et al. 2011, A\& $A$, 525, A103

Dale, D. A. \& Helou, G. 2002, ApJ, 576, 159

Désert, F.-X., Boulanger, F., \& Puget, J. L. 1990, A\&A, 237, 215

Dobbs, C. L., Theis, C., Pringle, J. E., \& Bate, M. R. 2010, MNRAS, 403, 625

Draine, B. T. \& Li, A. 2007, ApJ, 657, 810

Feldmeier, J. J., Ciardullo, R., \& Jacoby, G. H. 1997, ApJ, 479, 231

Fioc, M. \& Rocca-Volmerange, B. 1997, A\& A, 326, 950

Helfer, T. T., Thornley, M. D., Regan, M. W., et al. 2003, ApJS, 145, 259

Jarrett, T. H., Chester, T., Cutri, R., Schneider, S. E., \& Huchra, J. P. 2003, AJ, 125, 525 
Kennicutt, R. C., Jr., Armus, L., Bendo, G., et al. 2003, PASP, 115, 928

Koda, J., Sawada, T., Wright, M. C. H., et al. 2011, ApJS, 193, 19

Kohno, K., Tosaki, T., Matsushita, S., et al. 2002, PASJ, 54, 541

Lee, J. H., Hwang, N., \& Lee, M. G. 2011, ApJ, 735, 75

Pei, Y. C. 1992, ApJ, 395, 130

Salo, H. \& Laurikainen, E. 2000, MNRAS, 319, 377

Thronson, H. A., Jr., Rubin, H., \& Ksir, A. 1991, MNRAS, 252, 550

Tikhonov, N. A., Galazutdinova, O. A., \& Tikhonov, E. N. 2009, Astron. Lett., 35, 599

\section{Discussion}

GALlagher: Do you have any idea about what is going on in the nucleus of NGC 5195? It is the most intense source in the system in Spitzer $24 \mu \mathrm{m}$ images, a stronger point source than the M51 nucleus.

Mentuch: The bright emission at both 24 and $10 \mu \mathrm{m}$ is consistent with the emission by dust predicted by the Draine \& Lee (2007) model corresponding to a very high interstellar radiation field and a relatively low $\mathrm{PAH}$ fraction. It appears not to be related to star formation.

Chackrabarti: Following up on Jay Gallagher's question - you see M51's companion in the B-band (which traces gas and new stars) and at $24 \mu \mathrm{m}$ (which traces hot dust). Could there be some star formation that's not apparent in H-alpha because it's obscured by dust?

Mentuch: The B-band emission is well fit by the SED with SFH parameters for a tau model + burst (implying an old stellar population plus a starburst $400 \mathrm{Myr}$ ago), so there is no additional emission in the band associated with recent (within $10 \mathrm{Myr}$ ) star-formation. The hot dust is due to the high interstellar radiation field in the galaxy, whose origin is uncertain, although M51b is very bright in X-ray emission, which may be a clue. 